\title{
A Multi-Agent System for Modelling the Spread of Lethal Wilt in Oil-Palm Plantations
}

\author{
Conor Fahy \\ Institute of Artificial Intelligence \\ De Montfort University \\ Leicester, UK \\ conor.fahy@dmu.ac.uk
}

\author{
Fabio Caraffini \\ Institute of Artificial Intelligence \\ De Montfort University \\ Leicester, UK \\ fabio.caraffini@dmu.ac.uk
}

\author{
Mario Gongora \\ Institute of Artificial Intelligence \\ De Montfort University \\ Leicester, UK \\ mgongora@dmu.ac.uk
}

\begin{abstract}
Lethal Wilt (Marchitez Letal) is a disease which affects Elaeis Guineensis, a plant used in the production of palm oil. The disease is increasingly common but the spatialdynamics of the infection spread remain poorly understood. It is particularly dangerous due to the speed at which it spreads and the speed at which infected plants show symptoms and die. Early identification, or even better, accurate prediction of areas at high risk of infection can slow the spread of the disease and limit crop waste. This study is based on data collected over a fiveyear period from an affected plantation in Colombia. The aim of the study is to analyse the collected data to better understand how the disease spreads and then to model the behaviour. Based on insights from the initial analysis a multi-agent-based system is proposed to model the pattern of infection. The model is comprised of two steps; first Kernel Density Estimation is used to create an estimation of the distribution from which newly infected plants are drawn and this density estimation is then used to direct agents on a biased-walk of the surrounding areas. Results show that the model can approximate the behaviour of the disease and can predict areas which are at high risk of future infection.
\end{abstract}

Index Terms-Lethal Wilt, Palm Oil, Multi-Agent System, Disease Modelling

\section{INTRODUCTION}

Worldwide, the palm oil industry leads the supply of oils and fats within a very dynamic sector. Palm oil is formed from the mechanical pressing of the mesocarp of the palm fruit Elaeis guineensis [1]. The applications of this oil are extremely diverse, from cooking to cleaning products, greases and lubricants, the production of biodiesel and electrical energy [2], alongside uses in the leather, steel and textile industries. It is estimated that one hectare planted with the most common palm, i.e. Elaeis Guineensis, produces between 6 to 10 times more oil than any other type of oil-seed, and is second only to soybean oil [3]. One of the highest producers in South America is Colombia. The Colombian Government promote it as one of the main agricultural substitutes for illegal crops, leading to rural job creation and ultimately to a sustainable peace process. Annually, Colombia produces 1.6 million tonnes of palm oil (2\% of global production) [4]. However, successful and sustainable production is increasingly affected by a relatively new, highly infectious disease known

This research received funds from De Montfort University (Fabio Caraffini's VC2020 Additional Funding 2018/19)

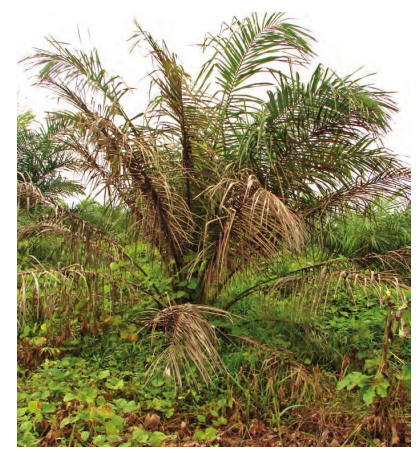

(a) Infected palms

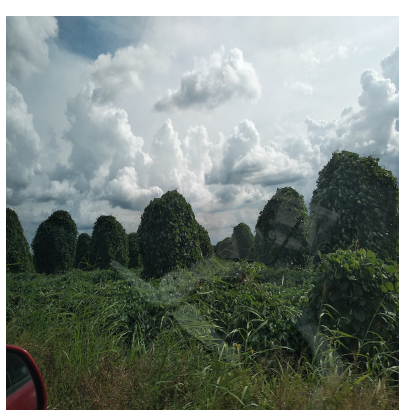

(b) Eradicated area
Fig. 1. Lethal Wilt symptoms (a), large areas of infected plants now desert after eradication (b). More photographs are available in [8]

as Lethal Wilt (Marchitez Letal in Spanish). The cause of the disease is unknown but is believed to be a phytoplasma, possibly related to the 'fatal yellowing' disease which is known to infect other species of palm plant [5]. Phytoplasmas are bacterial parasites in plant tissue and it is believed that insects such as plant-hoppers are responsible for their plantto-plant transmission. Phytoplasmas have been associated with diseases in several hundred plant species [7].

Symptoms of Lethal Wilt include the discolouration (yellowing) of leaves in mature plants (as shown in Figure 1 (a)). This is followed by leaf drying, tissue necrosis, leaf wilt, and eventually plant collapse. Plants showing these symptoms are injected with chemicals and destroyed, leaving large swaths of a plantation dormant (the aftermath of this culling is shown in Figure 1 (b)). Lethal Wilt is very destructive because it rapidly spreads through plantations and kills plants within 4-6 months after symptoms first appear [9].

This study is based on data collected from the Santa Barbara plantation in Colombia. The 2,800 hectare plantation is one of two plantations owned by Unipalmas [10] a company that is located in the Meta province ( $4^{\circ} 13^{\prime} 33^{\prime \prime} \mathrm{N}, 73^{\circ} 14^{\prime} 50^{\prime} \mathrm{W}$ ). The data is collected over a five year period from January 2013 to January 2018 and describes 258, 046 plants. The dataset contains the spatial position of each plant in the plantation, whether or not the plant displays Lethal Wilt symptoms, and an associated timestamp (month and year). 
The aim of this study is two-fold. One, to understand the pattern (if any) of the disease spread over time and two, to model this behaviour. Modelling the disease allows us to make predictions and identify areas which are at high risk of future infection.

We analyse the collected data and use the resultant insights to justify and inform a multi-agent-based system for modelling the behaviour of Lethal Wilt over time. The model consists of two steps; first, at each time step $t_{i}$ we use Kernel Density Estimation to estimate the probability distribution from which each of the newly infected plants at $t_{i}$ is drawn. Then, we use this density function to bias or 'direct' a multi-agentbased local search, whereby agents begin each time-step at newly infected sites and perform a biased random walk. Areas which are visited most frequently by the multi-agent system are considered to be at a higher risk of infection at $t_{i+n}$

The remainder of this paper is outlined as follows: Section II outlines relevant, related work. The data, analysis and developed model is described in Section III. The experimental set-up, metrics, and results are described in Section IV, and finally some conclusions along with future directions are described in Section V.

\section{RELATED WORK}

Palm Oil production is criticised for its negative environmental impact; deforestation, loss of biodiversity, and a disruption of the carbon cycle. However, this happens less often in Colombia than in other palm-oil producing countries. The reason for this is that between $60 \%$ and $70 \%$ of the palm oil is not produced on deforested land but on land which was formerly pasture land for livestock or land previously used to grow other crops [11]. However, there is still pressure to make the industry more sustainable. Governmental institutions, and international bodies, such as the United Nations Framework Convention on Climate Change (UNFCC) and the Round table on Sustainable Palm Oil (RSPO) demand that oil palm production should be managed responsibly and sustainably with minimal environmental impact. A study [12] on the academic response to these calls for greater sustainability show that the number of papers indexed in the Thomson Reuters Institute for Scientific Information has grown exponentially from just 4 in 2011 to 713 in 2013 and continues to grow. The study proposes a framework for palm oil sustainability and emphasises the necessity for greater collaboration between industry and academia. An exemplary study [13] uses system dynamics modelling to investigate the trade-off and synergies with different approaches to conserve biodiversity while improving people's livelihood, essentially the trade-off between development and conservation. Other modelling approaches are used to predict annual yield [14] and seasonal variation in yield [15], [16]. In [16] the authors propose OPRODSIM (Oil Palm Production Simulator) in order predict plantation responses to climatic changes during the first six years after planting. The simulation effectively simulates bunch yields and corroborates measured trends in soil water and crop water use.
Technological applications such as remote sensing are being used for land cover classification [17] and change detection [18]. After classifying palm-oil plantations from satellite data, change detection is used to monitor illegal expansion, or to identify whether new plantations have been developed on areas with a high conservation value (HCV). Perpetrators lose their sustainability certificate or receive heavy fines [19] and the data can inform local government law-making and regulations. Remote sensing is also used for tree counting [20], carbon estimation [21] and others [22], a comprehensive overview of remote sensing applications in palm oil production is given in [23]. A particularly relevant application is the use of remote sensing for disease detection [24], [25]. In [24] a portable hyperspectral instrument was used to differentiate healthy plants and plants infected with the disease Gandoderma boninsis before the infected plants display symptoms visible to the naked eye. The authors report a $97 \%$ prediction accuracy, however, the experiments were carried out in laboratory conditions and are not yet realistic for airborne (UAV) application due to the lower spatial resolution. In a more realistic large scale study using satellite images [25], infected plants can be identified with $62 \%$ accuracy, though only when the plants are at the terminal stage 4 of infection. Both studies focus on the Gandoderma boninsis disease and, to the best of our knowledge, no study exists for the Lethal Wilt disease, either using remote-sensing or in epidemiological models.

Epidemiological modelling can be classified into three types [26]. Descriptive models which provide hypotheses or experimental results but typically do not reveal the underlying mechanics of the disease progression. Predictive models (which are also descriptive) and Conceptual models which attempt to distinguish cause from effect and formalise the effect of specific events on the progression of the disease. In [27] thirteen challenges for modelling plant disease are outlined, these include factors such as multiple host species, accounting for time-varying infection rates, and the effects of vector preference on disease diffusion. As plant locations are fixed, modelling approaches are typically based on static graphs (nodes connected by edges) or on a lattice which can be regular [28] or Small World [29] (most nodes are not neighbours but can be reached via other nodes). With regular lattices, the probability of neighbouring nodes getting infected can be constant [30], or random [31] or can be treated as a function of distance, producing 'waves' in continuum models [32]. Agentbased disease models [33]-[35] also typically operate on a network or lattice. In [36] the Grapevine Leafroll Disease is modelled using heterogeneous agents (in age and infection state) with a cellular automaton system.

\section{Methodology}

In this section, we first analyse the dataset and based on insights from this analysis we propose a method to model the spread of Lethal Wilt. 


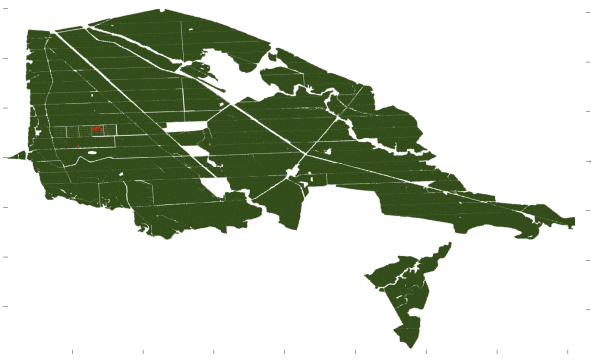

(a) Jan. 2013

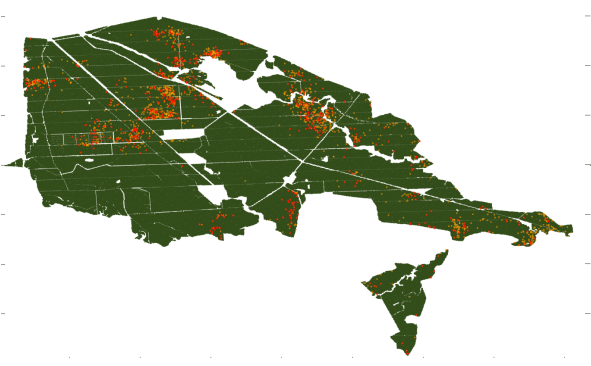

(b) July 2015

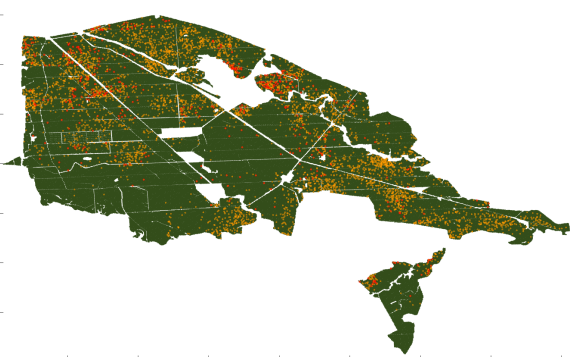

(c) Jan. 2018

Fig. 2. Spread of disease start month (a), mid-point (b), and final month (c). Red indicates plants infected in current month, orange indicates plants infected in previous six months.

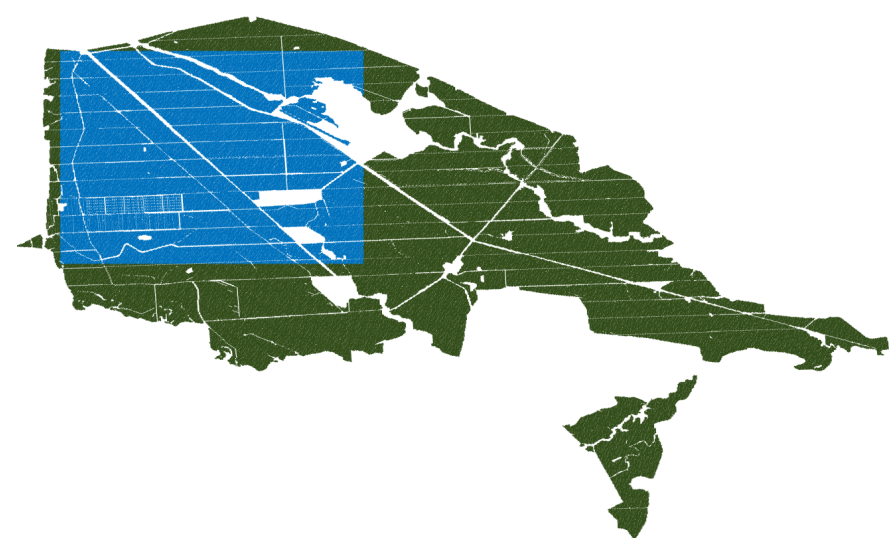

Fig. 3. Area considered in this study highlighted in blue

\section{A. Data Analysis}

The data were collected monthly between 2013 and 2018. The original data consists of 8 variables; 1) ID, 2) Block, 3) Parcel, 4) Line, 5) X-coordinate, 6) Y-coordinate, 7) Month, 8) Year. We extract variables 5-8; the $\mathrm{x}$ and $\mathrm{y}$ coordinate of the plant ( $\mathrm{x}$ and $\mathrm{y}$ in a coordinate system local to the plantation) and the timestamp for the sample. Each sample has an associated binary label; whether the plant exhibits Lethal Wilt symptoms or not. Before analysis, we remove incomplete samples and sort the data in time order from January 2013 to January 2018 (72 time-steps). This gives 258,046 samples with 93,345 infected plants (15\%) in total. The disease spread over time can be seen in Figure 2. Here, red marks represent plants infected in the current month, while the orange marks represent plants which have been infected within the previous 6 months. The figure shows the speed and range of infections over 5 years.

To simplify the preliminary data analysis we take a subset $S$ of the data (a square region of the map, displayed in Figure $3)$. This region contains 93,345 plants. We take this area and further divide it into $N * N$ grid cells. The analysis is performed on these grid cells at different granularities. Consider a cell $c_{i} \in S$ with 8 immediate neighbouring cells $c n_{j},\{j=1, \ldots, j=8\}$ and a time-window of $W$ months. We first analyse the frequency of the disease spreading locally
TABLE I

CELL RISK OF INFECTION AT VARYING GRANULARITIES ( WINDOWS $=6$ )

\begin{tabular}{cccccc}
\hline Grid & RI & PNI & ANI & NI & NNI \\
\hline $8^{2}$ & 0.94 & 0.83 & 0.99 & 0.56 & 0.04 \\
$16^{2}$ & 0.67 & 0.64 & 0.95 & 0.33 & 0.14 \\
$32^{2}$ & 0.4 & 0.38 & 0.71 & 0.15 & 0.08 \\
$64^{2}$ & 0.1 & 0.1 & 0.28 & 0.05 & 0.03 \\
\hline
\end{tabular}

TABLE II

CELL RISK OF INFECTION AT VARYING WINDOWS (GRANULARITY $=32^{2}$ )

\begin{tabular}{cccccc}
\hline Window & RI & PNI & ANI & NI & NNI \\
\hline 1 & 0.15 & 0.13 & 0.14 & 0.06 & 0.05 \\
3 & 0.28 & 0.27 & 0.54 & 0.11 & 0.07 \\
6 & 0.4 & 0.38 & 0.71 & 0.15 & 0.08 \\
12 & 0.57 & 0.53 & 0.86 & 0.02 & 0.08 \\
\hline
\end{tabular}

with the following questions; if a cell $c_{i}$ has shown signs of infection in the previous $W$ months, how often does it get re-infected in the subsequent $W$ months? (Re-Infection (RI)). If $c_{i}$ has been infected in the previous $W$ months, how frequently does a particular neighbouring cell $c n_{j}$ get infected in the subsequent $W$ months? (Particular Neighbour Infected (PNI)). If $c_{i}$ has been infected in the previous $W$ months, how frequently does at least one of its immediate neighbours get infected in the subsequent $W$ months? (Any Neighbour Infected (ANI)). We then analyse the frequencies of the disease appearing in previously uninfected areas; if $c_{i}$ has not been infected in the previous $W$ months, how often does it get infected in the subsequent $\mathrm{W}$ months? (New Infection (NI)). And finally, if $c_{i}$ has not been infected and neither have any of its immediate neighbours, how often does $c_{i}$ show signs of infection in the subsequent $W$ months? (Neighbours New Infection (NNI)).

The results, at different grid-granularities are displayed in Table I and with different values for $\mathrm{W}$ are displayed in Table II. The results are consistent and relative at each granularity and window size. If we take, for example, a $32^{2}$ grid and a 6 -month time-window we can say that if a cell has been infected in the previous 6 months then $40 \%$ of the time it will get re-infected in the following 6 months. We can say that any 
particular neighbouring cell (top left, right, etc.) has a similar, though slightly lower chance $(38 \%)$, of getting infected, and we can say that there is a very high chance $(70 \%)$ of at least one neighbouring cell getting infected. Conversely, if a cell has shown no signs of infection in the previous 6 months there is only a $15 \%$ chance of the cell getting infected, and if the cell or any of its immediate neighbours have not been infected there is a relatively small $(8 \%)$ chance of infection in the next months.

Based on this analysis we conclude that the disease is much more likely to spread locally from infected areas. This also highlights the inherent complexity of the problem; the disease is just as likely to spread to any of the neighbouring areas and the direction and severity of infection emerge as a consequence of the number of surrounding infected areas. These areas are themselves affected by their own surroundings.

\section{B. Proposed Method}

To model the spread of Lethal Wilt we build upon some of the insights from the previous data analysis. Ideally, the model will be biased towards the spatial and temporal locality of the infection spread. The model should also incorporate some of the apparent randomnesses of new infections and it should account for the complexity and emergent behaviour of the spread.

We propose a multi-agent based approach whereby agents take a directed walk from all newly infected cells. Based on the cells where these agents end their walk, coupled with the number of times each cell is visited during a walk, we make predictions of how the disease will spread.

There are two stages to the proposed model. First, Kernel Density Estimation (KDE) is performed. Areas with more infections have a higher density, this creates a compact representation of the problem and is used to direct each agent's walk. Each agent is biased to walk 'uphill' towards denser areas.

1) Kernel Density Estimation: A density estimator takes data, in this case the infected plants at time-step $t$ in the $N * N$ grid $S$, and produces an estimate of the probability distribution from which that data is drawn from. KDE is a non-parametric estimator and all the data points are used to make an estimate. A kernel is centred on each point in the data and the estimator smooths each observation over the local neighbourhood of that data point. The amount that point $x(i)$ contributes to the estimate at some point $x$ depends on the difference between $x(i)$ and $x$. This contribution is dependant on the choice of kernel function $K$ and the bandwidth (the width of the function) $h$. The estimated density of any point $x$ is described by:

$$
\hat{f}(x)=\frac{1}{N} \sum_{i=1}^{n} K\left(\frac{x-x(i)}{h}\right)
$$

We use a Gaussian Kernel $\left(\frac{1}{\sqrt{2 \pi}} \exp \left(-\frac{1}{2} \mu^{2}\right)\right)$. Bandwidth selection is a common problem, too small a value for $h$ can give a 'spikey' estimate (not enough smoothing) while large values for $h$ can over-smooth the estimate. This value can be tuned [37], or the AMISE (Asymptotic Mean Integrated Square Error) can be used [38]. This also needs to be estimated from the data, so $h$ is an estimate of an asymptotic approximation. We use the 'plug-in bandwidth selection method' outlined in [39] which is also non-parametric and data-driven. Here, $h$ is asymptotically optimal in the mean-squared error sense.

At the end of this step, the distribution of infection densities is estimated at time-step $t_{i}$. These densities are visualised $\mathrm{n}$ Figure 4.

2) Biased Walk: In the second part of the algorithm, agents start in newly infected cells and take steps number of steps. At each step of the walk, an agent can move into any of one of its immediate neighbouring cells $c n_{i}$. The probability of each cell being selected is proportional to its density. If $d_{i}$ is the density of a neighbouring cell, the probability of neighbouring cell $P_{c n_{i}}$ being selected is:

$$
P_{c n_{i}}=\frac{d_{i}}{\sum_{j=1}^{8} d_{j}}
$$

During each walk, if an agent visits a cell $c_{i}$, a counter for $c_{i}$ is incremented. Once all agents have completed their walks we have an $N * N$ matrix of predictions Pred, where each value in Pred represents the number of times an agent visited the corresponding cell in $S$. This allows us to visually display the model's prediction at $t_{i+W}$ using a heat map, where brighter areas represent the cells most visited by agents.

\section{EXPERIMENTAL SECTION}

In this section, we evaluate the model's performance both visually and analytically. Metrics are described along with the experimental set-up. Results and parameter sensitivity is discussed.

\section{A. Metrics and Set-Up}

We evaluate the performance in terms of True Positives (TP), True Negatives (TN), False Positives (FP), and False Negatives (FN). We evaluate the precision $P$ of the model :

$$
\mathrm{P}=\frac{\mathrm{TP}}{\mathrm{TP}+\mathrm{FP}}
$$

The model's recall score $R$ :

$$
\mathrm{R}=\frac{\mathrm{TP}}{\mathrm{TP}+\mathrm{FN}}
$$

And the Rand Index which is essentially the overall accuracy of the model :

$$
\mathrm{RI}=\frac{\mathrm{TP}+\mathrm{TN}}{\mathrm{TP}+\mathrm{FN}+\mathrm{TN}+\mathrm{FN}}
$$

In each experiment we start in the current time-step $t_{i}$ $\left(t_{1}=1, \ldots, t_{\text {end }}=(72-W)\right)$. We run the model and then evaluate the predictions against the ground-truth during the subsequent $W$ months. For example, with a 6-month window, the first run is in January 2013 and we evaluate the model's predictions against actual infections up to July 2013.

Due to the stochastic nature of the model, for each experiment we present the mean (and deviation) of 100 runs. 


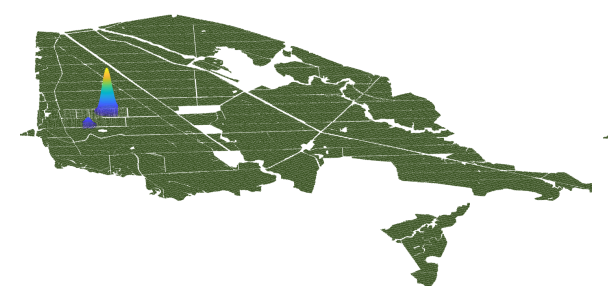

(a) Jan. 2013

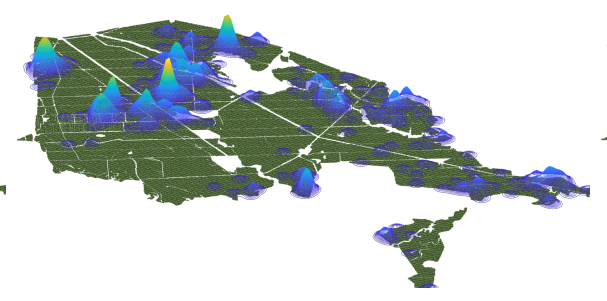

(b) July 2015

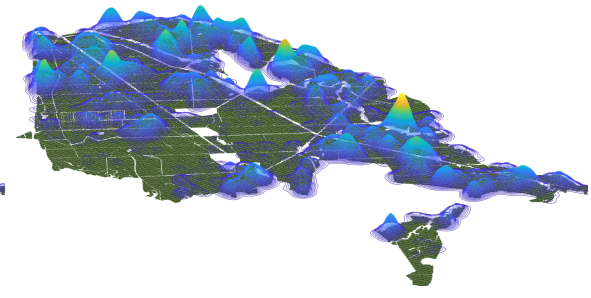

(c) Jan. 2018

Fig. 4. Density Estimation of infected plants over time. Start month (a), mid-point (b), and final month (c).

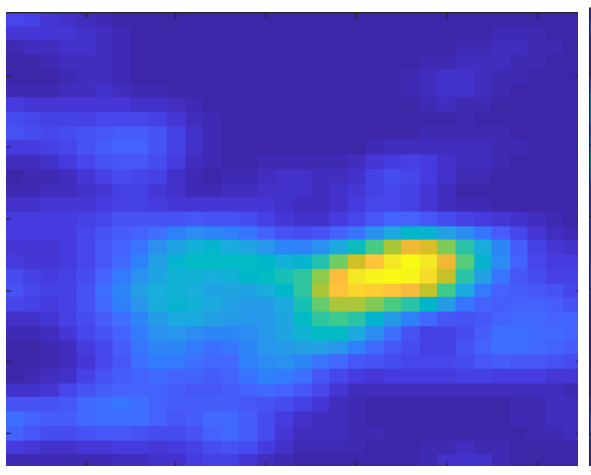

(a) Actual Density, July 2013

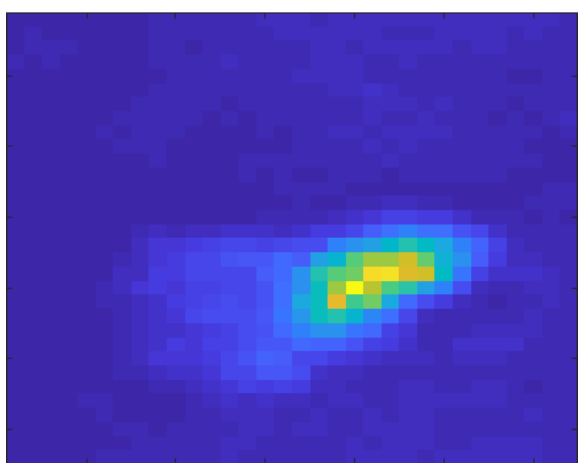

(d) Predicted Density for July 2013

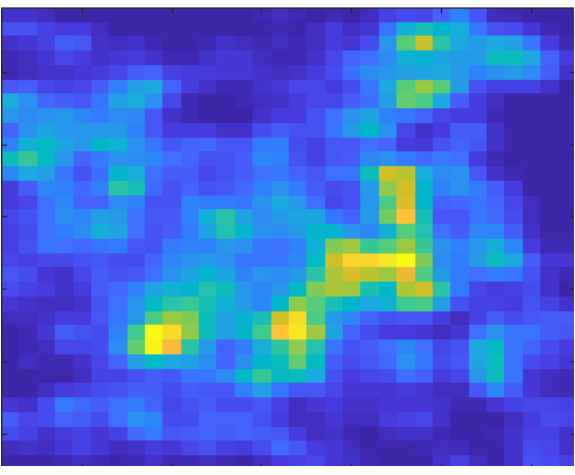

(b) Actual Density July 2015

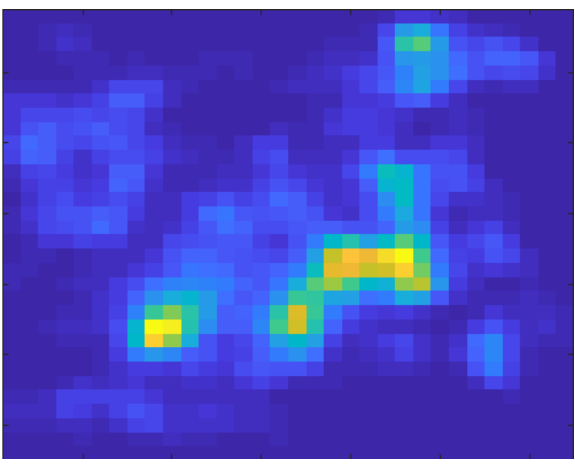

(e) Predicted Density for July 2015

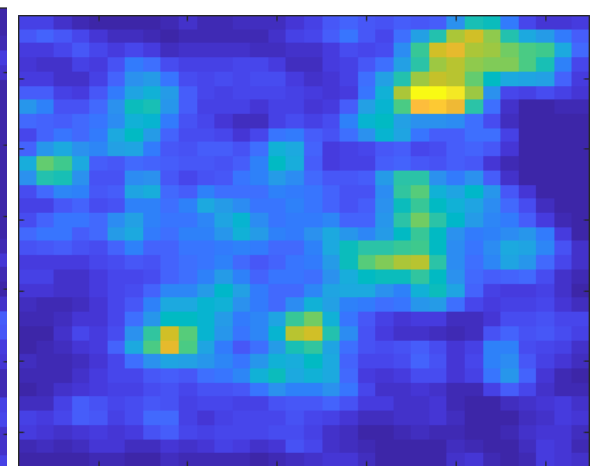

(c) Actual Density, Jan. 2018

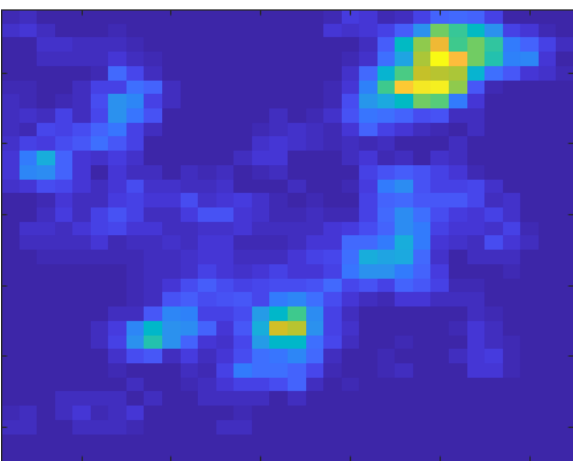

(f) Predicted Density for Jan. 2018

Fig. 5. $32^{2}$ grid with 6 month window. Top row displays actual densities. Bottom row displays the model's predicted densities (avg. 100 runs)

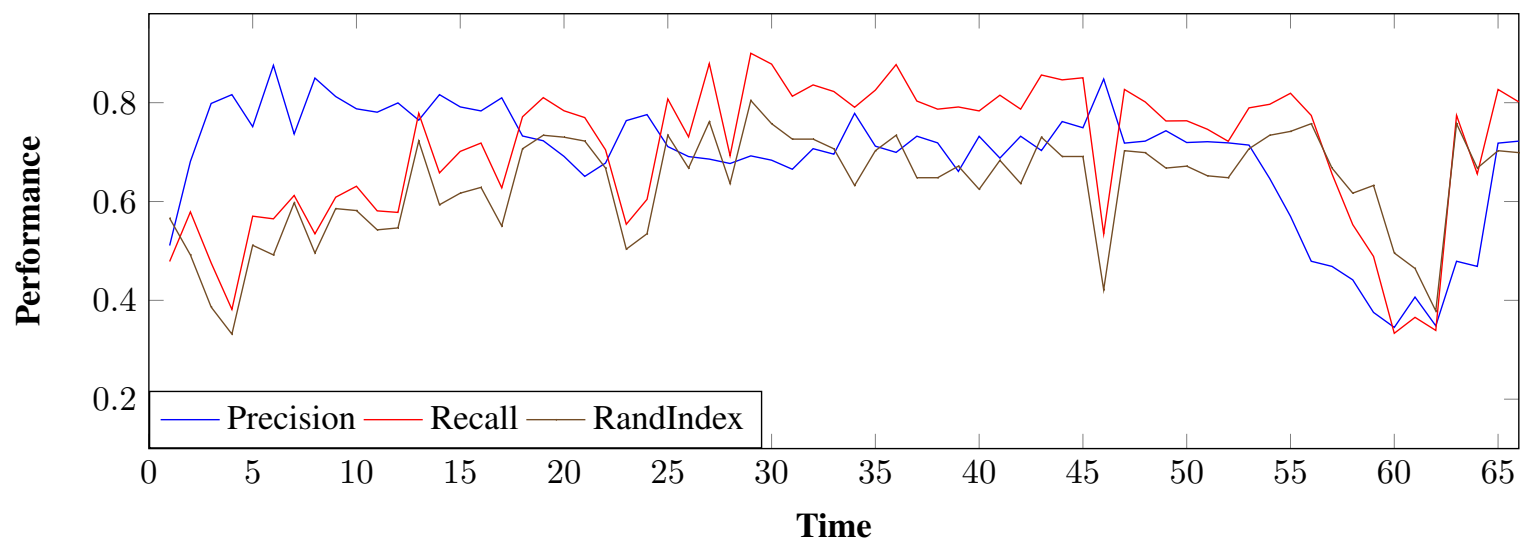

Fig. 6. Prediction accuracy each month, 100 agents take 3 steps (avg. 100 runs) 

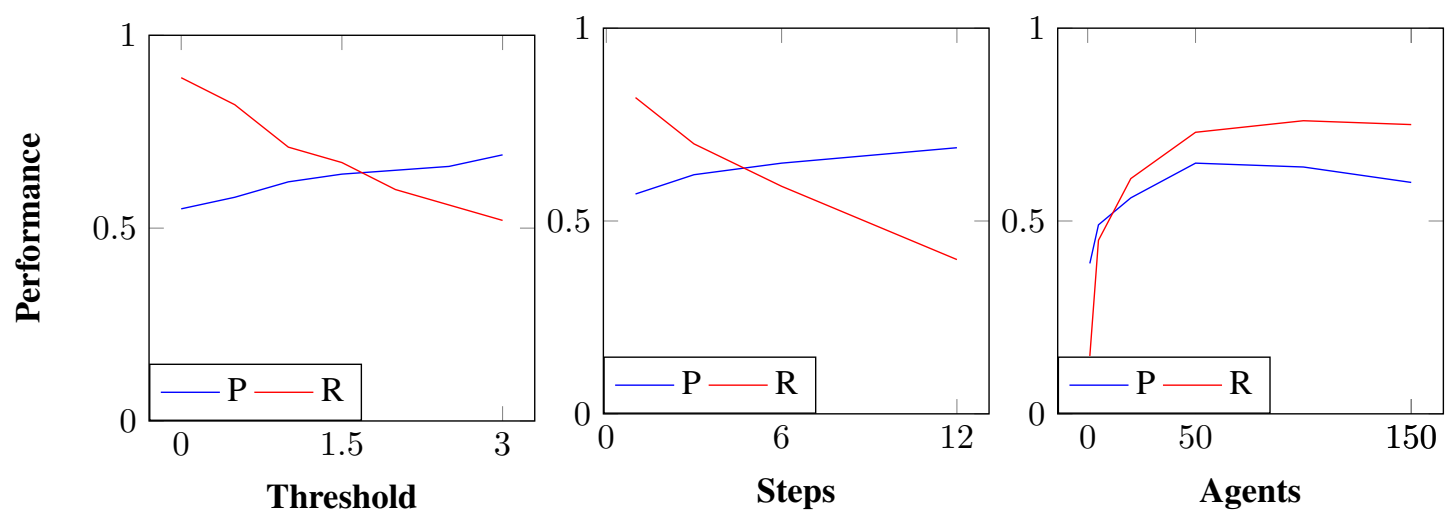

Fig. 7. Sensitivity of threshold (left) with 3 steps taken by 100 agents. Sensitivity of steps (middle) with 100 agents and a threshold of 1.5 , and number of agents (right) taking 3 steps with a threshold of 1.5. Performance measured in Precision (P) and Recall (R)

\section{B. Results}

Visual examples of the model's predictions are given in Figure 5. The results are based on a $32^{2}$ grid and show predicted densities 6 months in advance. The top row in the figure displays the actual densities at months 7 (6 months after the first prediction), month 33 (middle of the simulation) and month 72 (the final month of the simulation). In this figure, the brighter, yellow regions indicate a higher number of infections per cell. The bottom row displays the predicted densities for the same months. These densities are approximated by the number of times each cell was visited by an agent.

It can be seen that the model does not correctly predict every single new infection but can identify the major, concentrated areas of infection and approximates the spread of disease through the plantation.

To formally measure the model's performance we use the metrics described in the previous section. Here, we only count the cells where an agent finishes its walk (as opposed to each cell visited). If the predicted density of a cell is above a threshold value we assume that this is a positive prediction. For 60 agents taking 3 steps and a threshold value of 1.5 the model achieves $69 \%$ precision $( \pm 0.002), 75 \%$ Recall $( \pm 0.001)$ and a Rand Index of $74 \%( \pm 0.001)$. The month by month accuracy is displayed in Figure 5.

The sensitivity of each of these parameters is displayed in Figure 7 measured using the precision $(P)$ and recall $(R)$ scores. For the threshold and Steps parameters, it can be seen as expected that there is a trade-off between precision and recall. A lower threshold allows the model to make more predictions thus increasing the recall score, however, this results in a greater number of incorrect predictions which lowers the precision. Similarly with the number of steps, if an agent is allowed to take a lot of steps it will make more predictions but the confidence in each particular prediction will be lower (because fewer agents will land on the same cell if there is a greater range). This, coupled with the threshold value means that fewer predictions will be made. The number of agents does not greatly affect the model's performance if the number is sufficiently high $(>50)$. The model's performance is affected if this number is too low.

\section{Conclusion And Future Directions}

The study in this paper is based on data collected over five years from a palm-oil plantation in Colombia. Monthly data was collected from 2013 to 2018 and contains information about the infection rate and spread of Lethal Wilt (marchetez letal) disease throughout the plantation. A sub-sample of this data was used in this initial study $(\approx 95,500$ plants $)$ and the aim of the study was two-fold; to first analyse the collected data and attempt to understand how the disease spreads, and secondly, to model this pattern of infection.

Our analysis of the data shows that the disease is much more likely to spread to a local area (for example through insect vectors) rather than a far-way area (for example, via an airborne vector). Though, there still is a small chance that plants in previously unaffected areas can suddenly show symptoms. The direction and severity of the spread is an emergent consequence, a result of a local 'build-up' of infections. The data confirms the intuition, though modelling this is difficult due to the frequently stochastic behaviour of the process. We use the insights from the data analysis to build a model of the disease spread with the aim that the model should 1) conform to the spatial locality of infection spread (but allow for randomness), and 2) address the inherent complexity and the emergent behaviour of the spread. The proposed model is a multi-agent system which is comprised of two steps; first Kernel Density Estimation (KDE) is used to create an estimation of the distribution from which newly infected plants are drawn from and this density estimation is used to direct agents on a biased-walk on a $N * \times N$ grid. We say that the more frequently a particular cell is visited the higher its risk of future infection.

The resulting model approximates the spread of the disease and can predict concentrated areas of infection in advance along with the most likely direction of the spread. To formally gauge the model's performance we measure the precision, recall, and accuracy of the model's predictions with the ground 
truth. Over a 100 runs the model achieves an average score of $69 \%(0.002)$ precision, $75 \%(0.001)$ recall and $74 \%(0.001)$ accuracy.

Future work investigates more sophisticated agent-based models, for example, agents that can learn from previous decisions as opposed to a biased-walk directed only by an estimated density. In future work, the results will be tested on other areas of the plantation and scaled up to evaluate its performance across the entire plantation.

\section{REFERENCES}

[1] R. Corley, R. V. Hereward and Peter B.H. Tinker, "The oil palm, fifth edition" John Wiley and Sons December, 2015.

[2] D. Gromko "Tendrá éxito américa latina con el aceite de palma?" Latin American Science Org, 2015.

[3] R.M. Londono "Colombia está escribiendo una nueva historia de palmicultura sostenible" Boletin El Palmicultor, vol.1, num. 546, pp. 7-9, August, 2017.

[4] Food and Agriculture Organisation of the United Nations, www.fao.org/in-action/agronoticias/detail/en/c/1139169/ - accessed 27.01.2020

[5] E. Alvarez et al. "Candidatus Phytoplasma asteris' strains associated with oil palm lethal wilt in Colombia" Plant Disease, vol. 3, num. 98, pp. 311-318, 2014.

[6] Fedepalma, "Es possible controlar la marchitez letal si se aplican las recommendaciones de Cenipalma", Phytopathol, vol. 48, pp. 335-378, 2009.

[7] A. Bertaccini et. al " Metabolism of carbohydrates in oil palms (Elaeis guineensis Jacq.) affected by lethal wilt", Agonomy Colombia, num. 28, pp. 181-187, 2010.

[8] A. Pena, M. Gongora, I. Bonet, and F. Caraffini "Intelligent system to improve the sustainability of oil palm crops through the construction of forecasting maps" Figshare, https://doi.org/10.21253/DMU.11638095, January, 2020

[9] A.P. Perez and G. Cayon, "Metabolism of carbohydrates in oil palms (Elaeis guineensis Jacq.) affected by lethal wilt", Agronomy Colombia, num. 28, pp. 181-187, 2010.

[10] Unipalma, https://www.unipalma.com/

[11] J.C. Quezada, A. Etter, J. Ghazoul, A. Buttler and T. Guillaume, "Carbon neutral expansion of oil palm plantations in the Neotropics" Science Advances, num. 11, 2019.

[12] S.B Hansen, R. Padfield, K. Syayuti, S. Evers, Z. Zakariah, and S. Mastura, "Trends in global palm oil sustainability research", Journal of cleaner Production, num. 100, pp. 140-149, 2015.

[13] Collier at. al. "Science for action: the use of scoping models in conservation and development", Environmental science \& policy, vol. 14, num. 6, pp.628-638, 2011.

[14] P. Oettli, S.K. Behera, and T. Yamagata, "Climate based predictability of oil palm tree yield in Malaysia" Scientific reports, 8(1), pp.1-13, 2018

[15] I.E. Henson, "Modelling seasonal variation in oil palm bunch production using a spreadsheet programme" Journal of Oil Palm Research, vol. 17, p.27. 2005.

[16] I.E. Henson, Z. Yahya, M.R.M. Noor, M.H. Harun, and A.T. Mohammed, "Predicting soil water status, evapotranspiration, growth and yield of young oil palm in a seasonally dry region of Malaysia" Journal of Oil Palm Research, vol 19,pp.398-415, 2007.

[17] H. Z. M. Shafri and N. Hamdan, "Hyperspectral Imagery for Mapping Disease Infection in Oil Palm Plantation Using Vegetation Indices and Red Edge Techniques." American Journal of Applied Sciences vol. 6 num. 6, pp. 1031-1035. 2009.

[18] N. Wielaard, "Impact of Oil Palm Plantations on Peatland Conversion in Sarawak 2005-2010", edited by Summary Report: SarVision. 2011.

[19] K. T. Tan, K. T. Lee, A. R. Mohamed, and S. Bhatia, "Palm Oil: Addressing Issues and towards Sustainable Development." Renewable and Sustainable Energy Reviews vol 13, num. 2, pp. 420-427. 2009.

[20] H. Santoso, H. Tani, and X. Wang, "A Simple Method for Detection and Counting of Oil Palm Trees Using Highresolution Multispectral Satellite Imagery." International Journal of Remote Sensing vol. 37, num. 21, pp. 5122-5134. 2016
[21] L.K. Kho and M. R. Jepsen. "Carbon Stock of Oil Palm Plantations and Tropical Forests in Malaysia: A Review." Singapore Journal of Tropical Geography vol. 36, num. 2, pp. 249-266. 2015.

[22] K. C. Teng, J. Y. Koay, S. H. Tey, K. S. Lim, H. T. Ewe, and H. T. Chuah. "A Dense Medium Microwave Backscattering Model for the Remote Sensing of Oil Palm." IEEE Transactions on Geoscience and Remote Sensing, vol. 53 num. 6, pp. 3250-3259. 2014.

[23] K.L. Chong, K.D. Kanniah, C. Pohl, and K.P Tan, "A review of remote sensing applications for oil palm studies" Geo-spatial Information Science, vol. 20, num. 2, pp.184-200. 2017.

[24] H. Z. M. Shafri, N. Hamdan, and M. I. Saripan. "Semi-automatic Detection and Counting of Oil Palm Trees from High Spatial Resolution Airborne Imagery." International Journal of Remote Sensing, vol. 32 num. 8, pp. 2095-2115, 2011.

[25] H. Santoso, R.H. Gunawan, W. Jatmiko, W. Darmosarkoro, and B. Minasny. "Mapping and Identifying Basal Stem Rot Disease in Oil Palms in North Sumatra with QuickBird Imagery." Precision Agriculture vol 12, pp. 233-248. 2011

[26] J. Kranz and D.J. Royle, "Perspectives in mathematical modelling of plant disease epidemics" In: Scott PR and Bainbridge A (eds) Plant Disease Epidemiology Blackwell Scientific Publications, Oxford, London, Edinburgh, Melbourne, pp. 111-120, 1978.

[27] N.J. Cunniffe, B. Koskella, C.J.E. Metcalf, S. Parnell, T.R. Gottwald, and C.A. Gilligan, "Thirteen challenges in modelling plant diseases", Epidemics, vol. 10, pp.6-10, 2015.

[28] H. Hinrichsen, "Non-equilibrium critical phenomena and phase transitions into absorbing states" Advances in physics, vol. 49, num. 7, pp.815-958. 2000

[29] J. Marro and R. Dickman, "Nonequilibrium phase transitions in lattices models" Cambridge University Press, Cambridge , 1999.

[30] J. Filipe and M.M. Maule MM. "Effects of dispersal mechanisms on spatio-temporal development of epidemics" Journal of Theoretical Biology vol. 226, pp. 125-141. 2004.

[31] A. Barbour, D. Mollison, "Epidemics and random graphs" Stochastic processes in epidemic theory. New York, NY, USA: Springer, pp. 86-89, 1990.

[32] C.A. Russell, D.L. Smith, A. Waller, J.E. Childs, L.A. Real, "A priori prediction of disease invasion dynamics in a novel environment" Proceedings of the Royal Society of London, Series B: Biological Sciences, vol. 271, pp.21-25, 2004.

[33] M. Frasca, A. Buscarino, A. Rizzo, L. Fortuna, and S. Boccaletti," Dynamical network model of infective mobile agents" Physical Review E, vol. 74, num. 3, 2006.

[34] M.C. González and H.J. Herrmann, "Scaling of the propagation of epidemics in a system of mobile agents" Physica A: Statistical Mechanics and its Applications, vol. 340, pp.741-748. 2004.

[35] M. Jeger, M. Pautasso, O. Holdenrieder, and M.W. Shaw, "Modelling disease spread and control in networks: implications for plant sciences", New Phytologist, vol. 174, pp.279-297, 2007.

[36] S. S. Atallah, M.I. Gómez,J.M. Conrad, Jand J.P. Nyrop, "A plantlevel, spatial, bioeconomic model of plant disease diffusion and control: Grapevine leafroll disease" American Journal of Agricultural Economics, 97(1), pp.199-218. 2015

[37] J. Murillo and A.A. Rodríguez, "Algorithms for Gaussian bandwidth selection in kernel density estimators". Neural Information Systems, 2008.

[38] S.J. Sheather, "Density estimation". Statistical science, pp.588-597. 2004.

[39] Z. I. Botev, J.F. Grotowski, and D.P. Kroese, "Kernel density estimation via diffusion", The annals of Statistics, vol. 38, num. 5, pp.2916-2957, 2010. 